\title{
Eddy turbulence, the double mesopause, and the double layer of atomic oxygen
}

\author{
M. N. Vlasov and M. C. Kelley \\ School of Electrical and Computer Engineering, Cornell University, Ithaca, NY, USA \\ Correspondence to: M. C. Kelley (mck13@ cornell.edu)
}

Received: 27 April 2011 - Revised: 5 December 2011 - Accepted: 9 December 2011 - Published: 23 January 2012

\begin{abstract}
In this study, we consider the impact of eddy turbulence on temperature and atomic oxygen distribution when the peak of the temperature occurs in the upper mesosphere. A previous paper (Vlasov and Kelley, 2010) considered the simultaneous impact of eddy turbulence on temperature and atomic oxygen density and showed that eddy turbulence provides an effective mechanism to explain the cold summer and warm winter mesopause observed at high latitudes. Also, the prevalent role of eddy turbulence in this case removes the strong contradiction between seasonal variations of the $\mathrm{O}$ density distribution and the impact of upward/downward motion corresponding to adiabatic cooling/heating of oxygen atoms. Classically, there is a single minimum in the temperature profile marking the location of the mesopause. But often, a local maximum in the temperature is observed in the height range of $85-100 \mathrm{~km}$, creating the appearance of a double mesopause (Bills and Gardner, 1993; Yu and She, 1995; Gusev et al., 2006). Our results show that the relative temperature maximum in the upper mesosphere (and thus the double mesopause) can result from heating by eddy turbulence. According to our model, there is a close connection between the extra temperature peak in the mesosphere and the oxygen atom density distribution. The main feature of the $\mathrm{O}$ density height profile produced by eddy turbulence in our model is a double peak instead of a single peak of $\mathrm{O}$ density. A rocket experiment called TOMEX confirms these results (Hecht et al., 2004). Applying our model to the results of the TOMEX rocket campaign gives good agreement with both the temperature and oxygen profiles observed. Climatology of the midlatitude mesopause and green line emission shows that the double mesopause and the double layers of the green line emission, corresponding to the double $\mathrm{O}$ density height profile, are mainly observed in spring and fall (Yu and She, 1995; Liu and Shepherd, 2006). Further observations of the oxygen atom densities and the double mesopause would improve our understanding of the impact of turbulence on critical mesospheric parameters.
\end{abstract}

Keywords. Atmospheric composition and structure (Middle atmosphere - composition and chemistry) - Meteorology and atmospheric dynamics (Middle atmosphere dynamics; Turbulence)

\section{Introduction}

Neutral temperature measurements in the mesosphere and lower thermosphere (MLT) can be made using several different methods: $\mathrm{Na}, \mathrm{K}$, and $\mathrm{Fe}$ lidar measurements, radar tracking of inflatable falling spheres, and measurements of the $\mathrm{O}_{2}$ atmospheric bands and $\mathrm{CO}_{2}$ infrared emission. One of the curious features of the temperature height distribution is a relative maximum of the temperature in the upper mesosphere. This extra peak has been observed many times (Bills and Gardner, 1993; Yu and She, 1995; Gusev et al., 2006) and has been associated with a double mesopause. An example of the temperature height profile measured by $\mathrm{Yu}$ and She (1995) is shown in Fig. 1. According to the climatology of a midlatitude mesopause region inferred by $\mathrm{Yu}$ and She (1995), the double mesopause is observed in spring and fall.

The thermal balance of the mesosphere and lower thermosphere (MLT) is controlled by radiative heating due to absorption of solar $\mathrm{UV}$ radiation by $\mathrm{O}_{2}$ and $\mathrm{O}_{3}$, by chemical heating from exothermic reactions, by radiative cooling associated with infrared emission of $\mathrm{CO}_{2}$, and heating and cooling induced by dynamic processes (Brasseur and Solomon, 1986). The latter includes compression/expansion caused by downward/upward motion associated with the gravity wavedriven meridional circulation, as well as direct heating due to gravity wave dissipation and turbulent diffusion from breaking gravity waves. The Kelvin-Helmholtz instability (KHI), caused by sheared flow, can also contribute to eddy turbulence. Heating and cooling by the diurnal contraction and expansion of the MLT can also occur (for example, see Tohmatsu, 1990). 


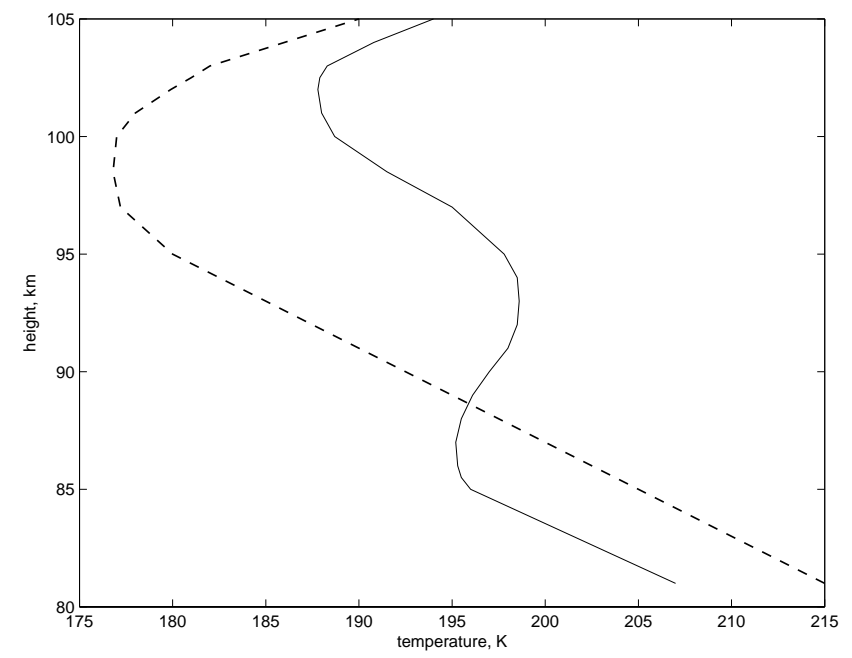

Fig. 1. Temperature height profiles observed by Yu and She (1995) (solid curve) and given by the MSISE-90 model (dashed curve).

It is well known that turbulence mixing of the main constituents provides the homosphere within which the mean molecular mass does not change and the height distributions of the main constituents have the same scale height (Banks and Kockarts, 1973). Atomic oxygen plays an important role in heating of the MLT due to energy released by exothermic three-body recombination of $\mathrm{O}$ atoms. This process is the most important chemical heating process in the upper mesosphere. Cooling by infrared radiation from $\mathrm{CO}_{2}$ also plays a very important role in the heat budget in the MLT. Thermal excitation of $\mathrm{CO}_{2}$ during collisions between $\mathrm{CO}_{2}$ and atomic oxygen is a source of this infrared radiation in the $15-\mu \mathrm{m}$ band. Localized cooling can also occur due to eddy transport in the presence of a temperature gradient (Gordiets and Kulikov, 1981; Vlasov and Kelley, 2010).

Atomic oxygen density is sensitive to transport by eddy turbulence and mass-averaged motion. In our previous paper (Vlasov and Kelley, 2010), we showed the effect of dynamic processes on the thermal balance of the upper mesosphere, together with the effect of these processes on atomic oxygen density. It was shown that seasonal variations of the $\mathrm{O}$ density and the altitude of the [O] peak, calculated with upward/downward motion corresponding to adiabatic cooling/heating, are opposite to the seasonal variations given by the MISIE-90 model (Hedin, 1991). The [O] transport due to eddy diffusion can eliminate this contradiction, and cooling/heating due to eddy heat transport and dissipation of turbulent energy is more important than adiabatic cooling/heating in explaining the cold summer and warm winter mesopause at high latitudes. Turbulence has been observed by a variety of techniques (see, for example, Hocking, 1990; Fukao et al., 1994; Kelley et al., 2003). The impact of eddy turbulence on the [O] density influences chemical heating and radiative cooling in the upper mesosphere and, at the

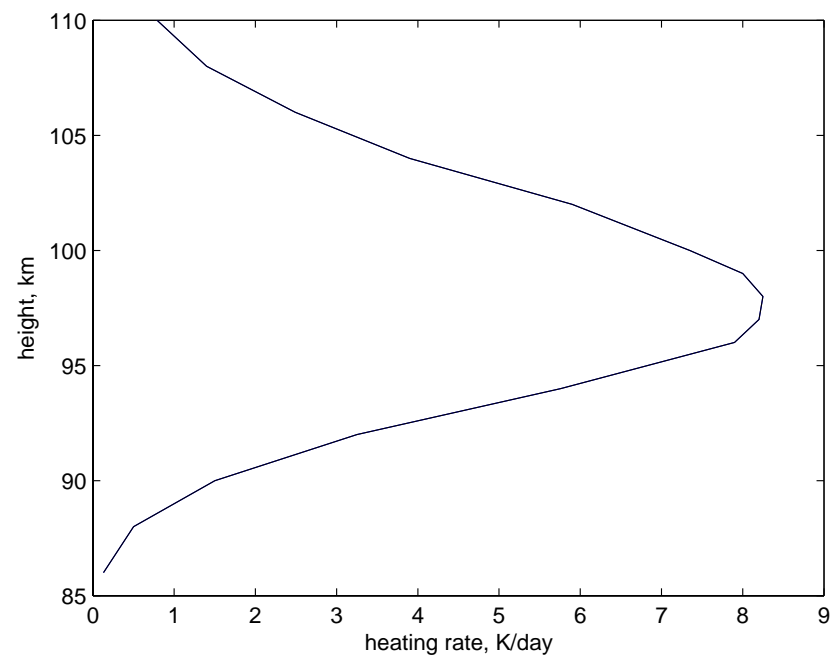

Fig. 2. Chemical heating in equinox due to atomic oxygen recombination.

same time, eddy turbulence can either cool or heat the mesosphere, depending on its height distribution.

The goal of this paper is to estimate the role of eddy turbulence in the production of a double mesopause and splitting of the atomic oxygen layer in the mesosphere because both phenomena are observed during the same period, namely, at equinox. We model the impact of this process on the temperature and atomic oxygen density profiles and compare the results to published experimental data. To our knowledge, this is the first attempt to relate the double mesopause to the atomic oxygen distribution.

\section{A summary of primary processes in the upper mesosphere}

Consider the primary processes in the normal mid-latitude MLT. Chemical heating plays an important role in the upper mesosphere due to the exothermic reaction

$\mathrm{O}+\mathrm{O}+\mathrm{M} \rightarrow \mathrm{O}_{2}+\mathrm{M}$

Height profiles of the heating rate of this reaction calculated using the neutral composition given by the MSISE-90 model in winter and summer are shown in Fig. 2. The maximum value of this heating estimated by Smith et al. (2003) is $12 \mathrm{~K} \mathrm{day}^{-1}$. Also, additional heating due to the exothermic reaction,

$\mathrm{H}+\mathrm{O}_{3} \rightarrow \mathrm{OH}+\mathrm{O}_{2}$

is important at night. Note in the upper mesosphere that the ozone density strongly depends on the $\mathrm{O}$ density according to the formula (Brasseur and Solomon, 1986),

$\left[\mathrm{O}_{3}\right]=\frac{k_{2}[\mathrm{M}]\left[\mathrm{O}_{2}\right][\mathrm{O}]}{J_{\mathrm{O}_{3}}+k_{3}[\mathrm{O}]} \approx \frac{k_{2}[\mathrm{M}]\left[\mathrm{O}_{2}\right][\mathrm{O}]}{J_{\mathrm{O}_{3}}}$ 
where $J_{\mathrm{O}_{3}}$ is the ozone photodissociation coefficient. The heat released by other chemical reactions given in Table 1 is small. Thus, atomic oxygen plays the most important role in chemical heating in the upper mesosphere.

Heating due to absorption of solar radiation by molecular oxygen and ozone occurs in the upper mesosphere. However, this heating is also smaller than chemical heating.

The main heat loss in the upper mesosphere is due to infrared radiation of $\mathrm{CO}_{2}$ in the $15-\mu \mathrm{m}$ band excited by the collision of $\mathrm{CO}_{2}$ with atomic oxygen:

$\mathrm{CO}_{2}+\mathrm{O} \rightarrow \mathrm{CO}_{2}\left(01^{1} 0\right)+\mathrm{O}$

Radiative de-excitation and radiation absorption must be taken into account. Radiation absorption in the $15-\mu \mathrm{m}$ band of $\mathrm{CO}_{2}$ may result in a considerable reduction in the radiative cooling rate at heights below $100 \mathrm{~km}$. We used a parameterization developed by Fomichev et al. (1993) to calculate cooling by this infrared radiation. In conclusion, atomic oxygen plays the most important role in heating and cooling of the upper mesosphere.

Eddy diffusion induces a downward flux of atomic oxygen below the turbopause. An increase in this flux causes a net decrease in atomic oxygen density because of atomic oxygen transport to the recombination region. Thus, the impact of eddy turbulence on thermal balance is not limited to eddy conductivity and dissipation of turbulence energy because eddy turbulence can also change chemical heating and radiative cooling induced by Reactions (R1), (R2), and (R3), respectively.

\section{Heating and cooling by eddy turbulence}

There are different approaches and numerical models for estimating the heating/cooling rates induced by gravity waves in the MLT (Medvedev and Klaassen, 2003; Becker, 2004; Akmaev, 2007; Becker and McLandress, 2009). All models start from the ubiquity of gravity waves and then calculate the heating/cooling corresponding to the different dynamic processes induced and driven by these waves. Some models estimate the eddy diffusion coefficient, as mentioned in the introduction. However, we start from eddy turbulence and try to estimate the corresponding heating/cooling rates. In this case, the heating/cooling rate of eddy turbulence is given by the formula (see, for example, Fritts and Luo, 1995),

$$
Q_{\mathrm{ed}}=\frac{\partial}{\partial z}\left[K_{\mathrm{ec}} C_{p} \rho\left(\frac{\partial T}{\partial z}+\frac{g}{C_{p}}\right)\right]+K_{\mathrm{ec}} \rho \frac{g}{T c}\left(\frac{\partial T}{\partial z}+\frac{g}{C_{p}}\right),
$$

where $K_{\text {ecc }}$ is the eddy heat conductivity, $\rho$ is the undisturbed gas density, $g$ is the gravitational acceleration, $T$ is the temperature, $C_{p}$ is the specific heat at constant pressure, and $c$ is a dimensionless constant commonly taken to be 0.8 (Lübken, 1997; Hocking, 1999). The first term on the right side of Eq. (2) is the heat flux divergence corresponding to
Table 1. Exothermic reactions and rate coefficients.

\begin{tabular}{ll}
\hline Reaction & Rate coefficient \\
\hline $\mathrm{O}+\mathrm{O}+\mathrm{M} \rightarrow \mathrm{O}_{2}+\mathrm{M}$ & $k_{1}=4.7 \times 10^{-33}(300 / T)^{2}$ \\
$\mathrm{O}+\mathrm{O}_{2}+\mathrm{M} \rightarrow \mathrm{O}_{3}+\mathrm{M}$ & $k_{2}=6 \times 10^{-34}(300 / T)^{2.4}$ \\
$\mathrm{O}+\mathrm{O}_{3} \rightarrow \mathrm{O}_{2}+\mathrm{O}_{2}$ & $k_{3}=8 \times 10^{-12} \exp (-2060 / T)$ \\
$\mathrm{H}+\mathrm{O}_{3} \rightarrow \mathrm{OH}+\mathrm{O}_{2}$ & $k_{4}=1.4 \times 10^{-10} \exp (-470 / T)$ \\
$\mathrm{O}+\mathrm{OH} \rightarrow \mathrm{O}_{2}+\mathrm{H}$ & $k_{5}=2.2 \times 10^{-11} \exp (120 / T)$ \\
$\mathrm{O}+\mathrm{HO}_{2} \rightarrow \mathrm{OH}+\mathrm{O}_{2}$ & $k_{6}=3 \times 10^{-11} \exp (200 / T)$ \\
\hline
\end{tabular}

$k_{1}$ and $k_{2}$ in units $\mathrm{cm}^{6} \mathrm{~s}^{-1} ; k_{3}-k_{6}$ in units $\mathrm{cm}^{3} \mathrm{~s}^{-1}$

heat transport. The second term is the turbulent energy dissipation rate initiated by the dynamic instability of gravity waves. Note, for example, that the first term presents the divergence of heat flux corresponding to the heat flux given by Becker (2004) for the parameter $P_{\text {reff }}=1$ and the heat flux given by Eq. (23) in Akmaev (2007). The second term is similar to the total wave energy disposition rate per unit mass, $\varepsilon=K \omega_{\mathrm{B}}^{2} / c$ (Weinstock, 1978), where $\omega_{\mathrm{B}}$ is the buoyancy frequency given by Eq. (5) and the parameter $c$ can be associated with the turbulent Prandtl number, $P$. Great debate exists about the value of the turbulent Prandtl number. First, this problem is due to different assumptions about gravity wave energy transport and dissipation and localized or uniform induced turbulence. However, this problem is not within the scope of this paper. We restrict our calculations of turbulent heating/cooling only to different values of $c$.

In addition, dynamic instability is induced by large vertical shears of horizontal wind that is not necessarily due to gravity waves,

$S=\left[(\partial u / \partial z)^{2}+(\partial v / \partial z)^{2}\right]^{1 / 2}$

where $u$ and $v$ are the zonal and meridional wind profiles. We emphasize that the peak temperature in the upper mesosphere is observed at altitudes above $90 \mathrm{~km}$ where shear becomes very important in the production of turbulence (Larsen, 2002). Dynamic instability is characterized by the Richardson number, defined as

$R i=\frac{\omega_{\mathrm{B}}^{2}}{S^{2}}$

where

$\omega_{\mathrm{B}}^{2}=\frac{g}{T}\left(\frac{\partial T}{\partial z}+\frac{g}{C_{p}}\right)$.

The atmosphere is considered to be dynamically unstable when $0<R i<1 / 4$ (Peixoto and Oort, 1992). Note that the positive temperature gradient needed for the temperature peak in the upper mesosphere increases the buoyancy frequency and the $R i$ value. Large wind shears are necessary for small $R i$ and are needed to support dynamic instability 


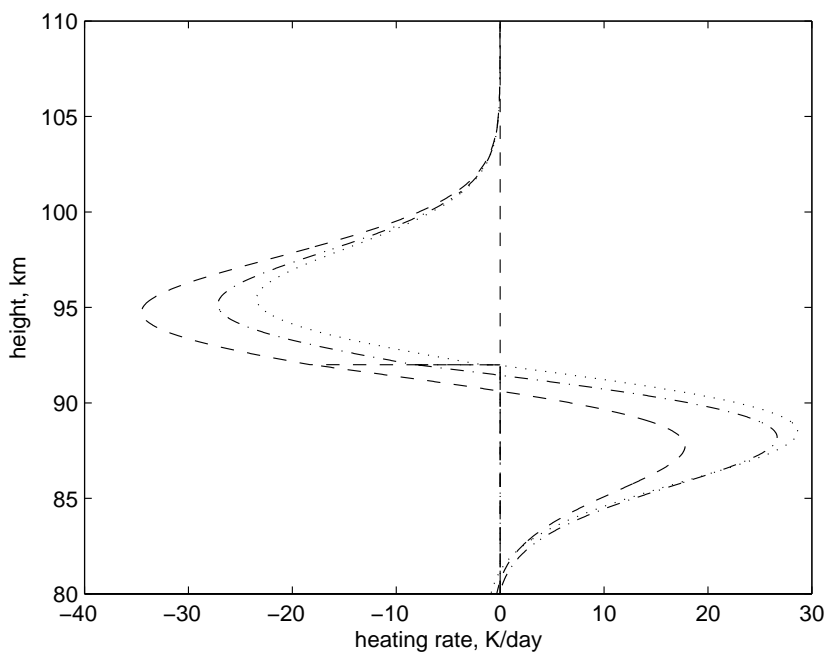

Fig. 3. Heating by eddy turbulence calculated with $R i=0.8$ (dashed line), $R i=0.4$ (dashed-dotted line), and $R i=0.3$ (dotted line) plus other parameters: $K_{\mathrm{ec}}^{0}=2 \times 10^{4} \mathrm{~cm}^{2} \mathrm{~s}^{-1}, K_{\mathrm{ec}}^{m}=2 \times 10^{6} \mathrm{~cm}^{2} \mathrm{~s}^{-1}$, $S_{1}=0.02 \mathrm{~km}^{-1}, S_{2}=0.04 \mathrm{~km}^{-1}, S_{3}=0.02 \mathrm{~km}^{-2}, T_{0}=211 \mathrm{~K}$. The vertical line shows the $K_{\text {ec }}$ peak altitude.

during the double mesopause. Note in our case that $c=R f_{0}$ is the dynamic Richardson number statistically steady turbulent motion, $R f_{0}=R i / P$.

Consider the heating/cooling due to eddy turbulence in detail. The altitude profile of $K_{\mathrm{ec}}$ is given by the widely used approximation suggested by Shimazaki (1971):

$$
\begin{aligned}
K_{\mathrm{ec}} & =K_{\mathrm{ec}}^{0} \exp \left[S_{1}\left(z-z_{m}\right)\right]+\left(K_{\mathrm{ec}}^{m}-K_{\mathrm{ec}}^{0}\right) \exp \left[-S_{2}\left(z-z_{m}\right)\right]^{2} \\
z & <z_{m} \\
K_{\mathrm{ec}} & =K_{\mathrm{ec}}^{m} \exp \left[-S_{3}\left(z-z_{m}\right)^{2}\right] \quad z>z_{m}
\end{aligned}
$$

where $S_{1}, S_{2}$, and $S_{3}$ are reciprocals of the scale heights, $z_{m}$ is the height of the $K_{\mathrm{ec}}$ peak, and $K_{\mathrm{ec}}^{0}$ and $K_{\mathrm{ec}}^{m}$ are values at the low boundary and in the peak, respectively.

The total heating/cooling due to both dissipation of the turbulent energy and to eddy heat conduction is shown in Fig. 3. These values are calculated using the mean temperature profile with $T_{0}=211 \mathrm{~K}$ at $80 \mathrm{~km}$. The heating peak is located at about 5-6 km below the $K_{\text {ec }}$ peak. Note that heat conductivity corresponding to the first term on the right side of Eq. (2) can heat or cool the mesosphere. Cooling dominates at high altitudes where the $K_{\text {ec }}$ gradient (Eq. 2) is negative and heating dominates below the $K_{\text {ec }}$ peak where the gradient is positive. Heating/cooling strongly depends on the value of $c$, which is equal to the $R i$ value for a Prandtl number equal to 1 (uniform turbulence). We will consider this problem below.

We now consider the experimentally determined mean temperature profiles having double temperature minima as constructed by $\mathrm{Yu}$ and She (1995) using three years of lidar data taken over Fort Collins, Colorado. According to the data, double mesopauses are observed in spring and fall and

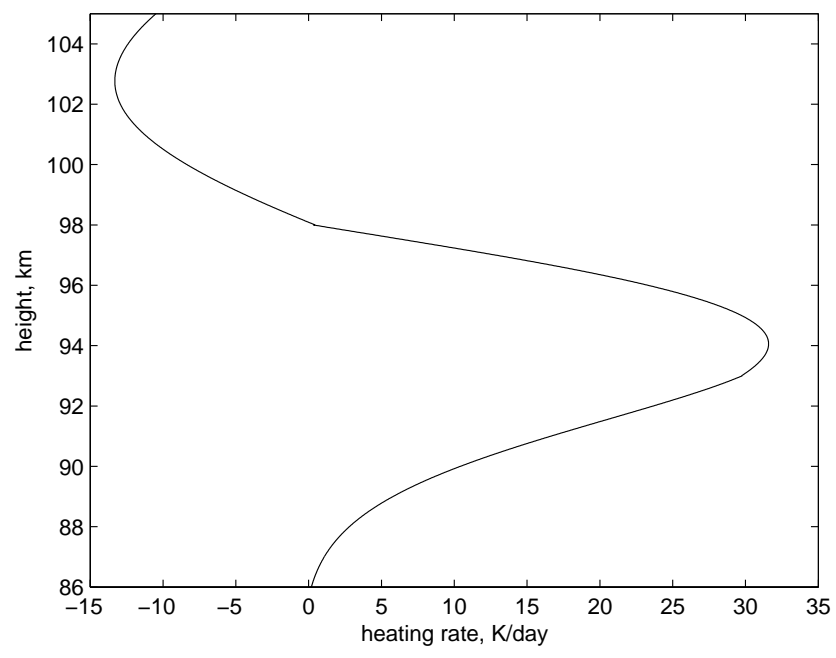

Fig. 4. Heating rate profile corresponding to the double mesopause shown in Fig. 1 and calculated with $R i=0.5$ and $P=1$.

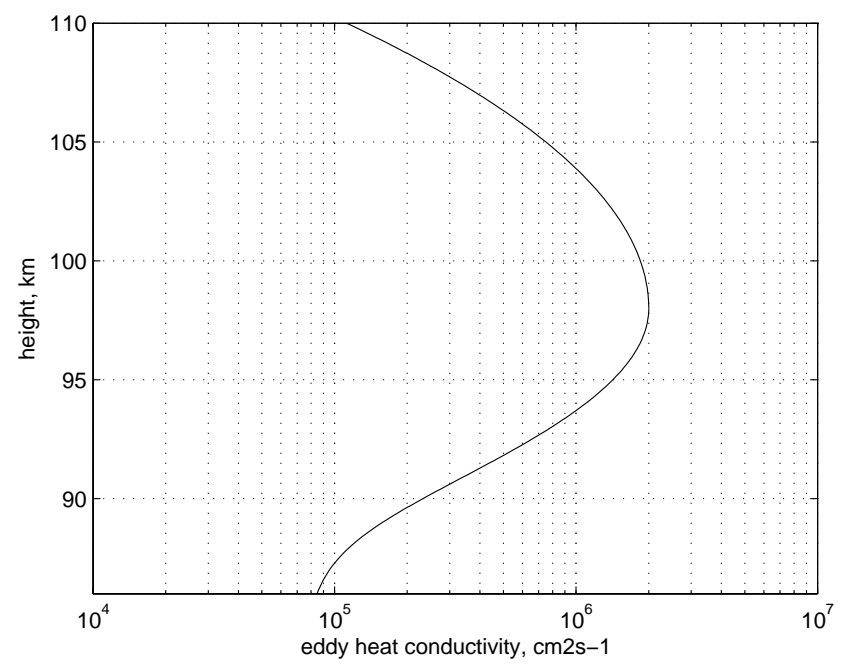

Fig. 5. The eddy heat conductivity coefficient profile used to calculate the heating rate profile shown in Fig. 4. This is based on eddy diffusivity and taking $P=1$.

are less common in summer and winter. A mean temperature profile with a double mesopause in March is shown in Fig. 1 (Yu and She, 1995). The height profiles given by models MSIS-90 and CIRA-86 are also shown. The measured temperatures between 90 to $100 \mathrm{~km}$ are higher than the temperatures given by empirical models. The heating rate due to eddy turbulence needed to explain the mean temperature profile given by $\mathrm{Yu}$ and She (1995) is shown in Fig. 4. The $K_{\text {ec }}$ height distribution is shown in Fig. 5.

As seen from Figs. 4 and 5, the heating peak induced by turbulence is located below the $K_{\text {ec }}$ peak altitude. Note that the $K_{\text {ec }}$ altitude is very close to the wind shear peak (Larsen, 2002). 


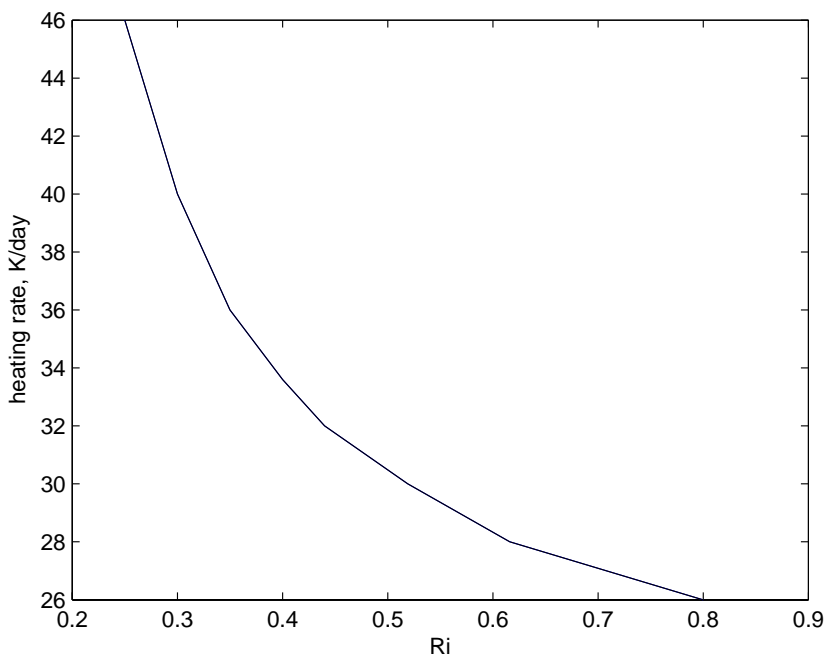

Fig. 6. Dependence of the maximum heating rate on the Richardson number for the eddy diffusion shown in Fig. 5 and corresponding to conditions for the heating rate shown in Fig. 4.

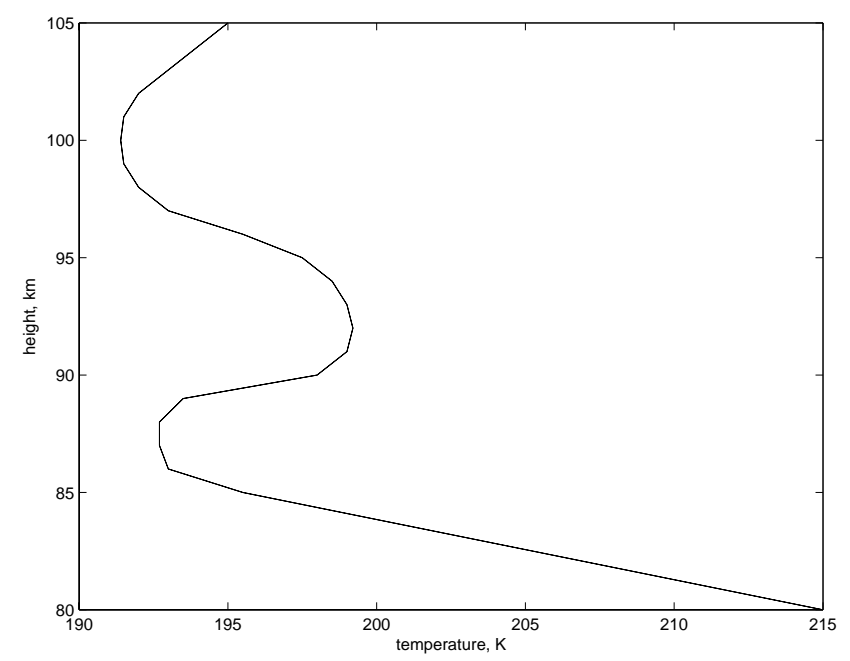

Fig. 7. Temperature height profile calculated with the heating rate shown in Fig. 4 and the other processes discussed in Sect. 2.

As seen from Fig. 6, the eddy turbulence heating significantly depends on the Richardson number for uniform turbulence, $P=1$. This means that the eddy heat conductivity needed to produce the temperature peak observed in the upper mesosphere decreases with decreasing $R i$.

As seen from Fig. 5, the eddy heat conductivity peak corresponding to this heating is located at $98 \mathrm{~km}$ with a $K_{\mathrm{ec}} \max -$ imum value of $2 \times 10^{6} \mathrm{~cm}^{2} \mathrm{~s}^{-1}$. This $K_{\text {ec }}$ value is less by a factor of 3 than the value estimated by Gordiets and Kulikov (1981) and Vlasov and Korobeynikova (1991) as the lower limit of the $K_{\text {ec }}$ peak value for producing a temperature peak in the upper mesosphere. According to their modeling, the temperature profile significantly differs from the usual pro-

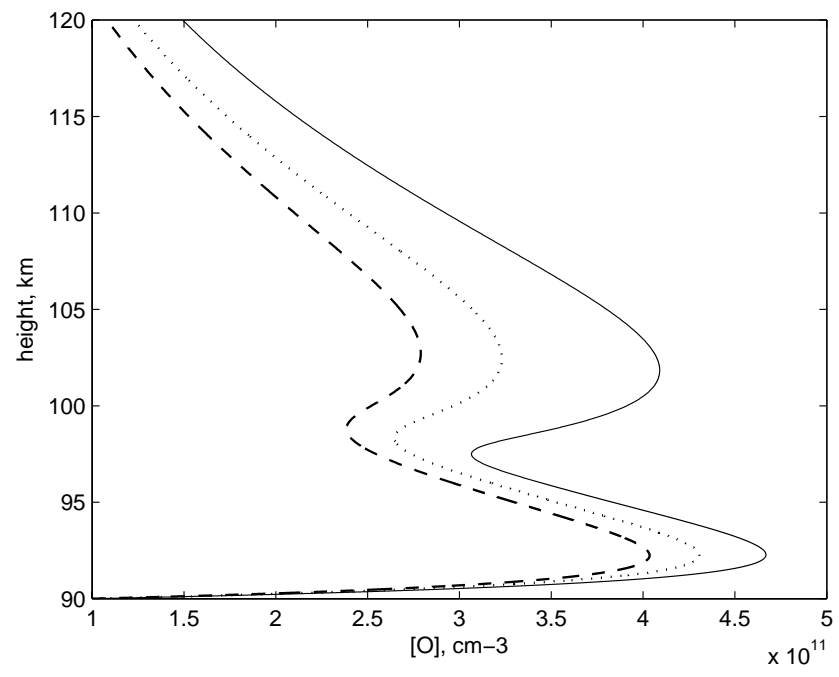

Fig. 8. The [O] height profile shown by the lowest curve calculated with the eddy diffusion coefficient shown in Fig. 5. The profile shown by the middle and upper curves corresponds to calculations with $S_{3}$ increased by a factor of 2 and 4 , respectively.

file if the eddy diffusion coefficient exceeds $6 \times 10^{6} \mathrm{~cm}^{2} \mathrm{~s}^{-1}$.

The temperature height profile calculated with the heating/cooling rates shown in Fig. 4, together with the other less important processes discussed in Sect. 2, is shown in Fig. 7. This profile is in good agreement with the mean measured profile shown in Fig. 1.

Note that the term "eddy heat conductivity" corresponds to the diffusive heat transport, which coincides with the eddy momentum transport for $P=1$ because $P=K_{\text {ed }} / K_{\text {ec }}$ (for example, see Gordiets and Kulikov, 1981). In this case, we can use the eddy diffusion coefficient, $K_{\text {ed }}$, equal to the eddy heat conductivity for calculating the atomic oxygen distribution. The [O] height profile calculated with this $K_{\text {ed }}$ is shown by the dashed curve in Fig. 8. This [O] profile has two peaks. The upper peak is mainly produced by eddy and molecular diffusion and the lower peak is produced by photochemistry and eddy diffusion. Vlasov and Davydov (1993) first showed that a height profile of atomic oxygen with two peaks could be induced by eddy and molecular diffusion together with photochemistry. As seen from the [O] height profiles shown in Fig. 8, the splitting depth of the [O] layer strongly depends on the value $S_{3}$, which characterizes the negative gradient of the eddy diffusion coefficient above the $K_{\text {ec }}$ peak. However, a steep negative gradient induces strong cooling above the temperature peak in the upper mesosphere. The simultaneous measurements of the temperature peaks and the [O] double layers are needed to make future progress.

Emission height profiles with two peaks radiated by atomic oxygen were observed by WINDII on UARS (Liu and Shepherd, 2006) and The Turbulent Oxygen Mixing Experiment (TOMEX) (Hecht et al., 2004). TOMEX combined rocket and $\mathrm{Na}$ lidar measurements of mesospheric 


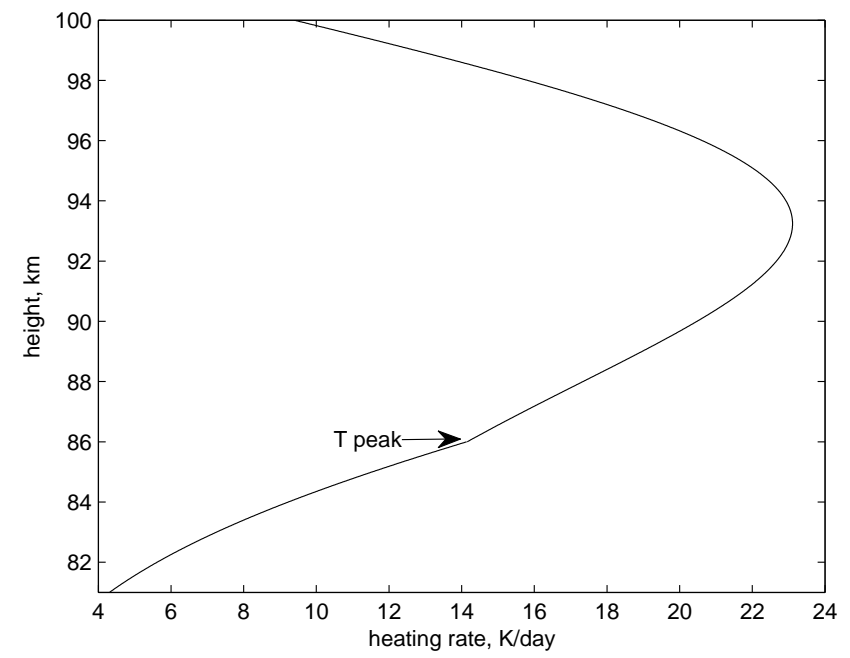

Fig. 9. Height profile of the heating rate calculated with the $K_{\mathrm{ed}}$ height distribution from the appropriate TIME-GCM model for TOMEX (Hecht et al., 2004). The altitude of the $T$ peak is shown by the arrow.

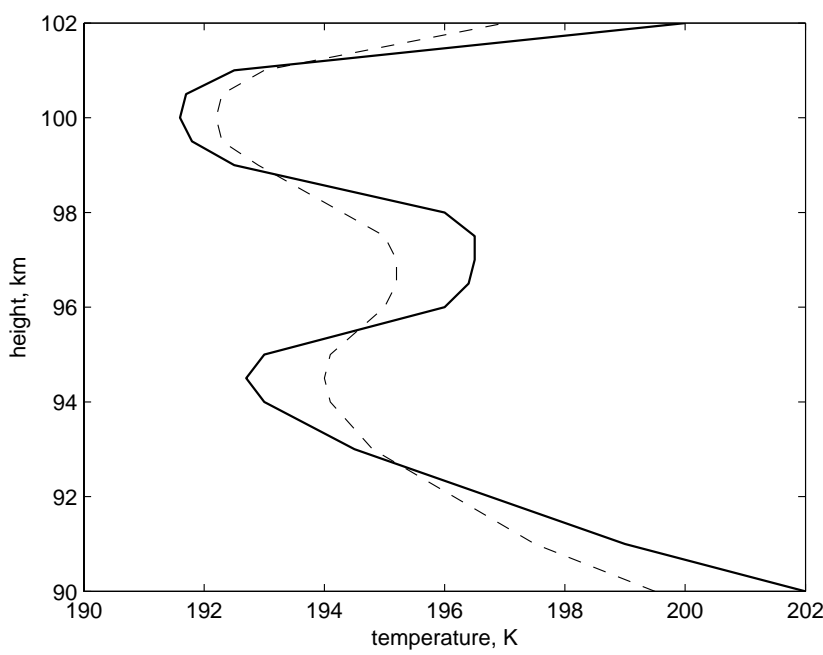

Fig. 10. Temperature height profiles measured by the TOMEX lidar (solid line) (Hecht et al., 2004) and calculated by the model (dashed line).

parameters. The temperature peak of about $230 \mathrm{~K}$ was measured at approximately $85 \mathrm{~km}$ altitude using a sodium lidar. According to the TIME-GCM model, the eddy heat conductivity coefficient corresponding to the calculated temperature with an $86 \mathrm{~km}$ peak has a maximum at $98 \mathrm{~km}$ altitude with a maximum value of $2.5 \times 10^{6} \mathrm{~cm}^{2} \mathrm{~s}^{-1}$. Using this $K_{\text {ec }}$ profile and the temperature profile with an $86 \mathrm{~km}$ peak, we can calculate the heating rates and height profiles of these rates as shown in Fig. 9. The heating maximum is at $93 \mathrm{~km}$ altitude, an altitude significantly higher than the temperature maximum altitude given by the TIME-GCM model. According to our model (see, for example, Figs. 1 and 4), the tempera-

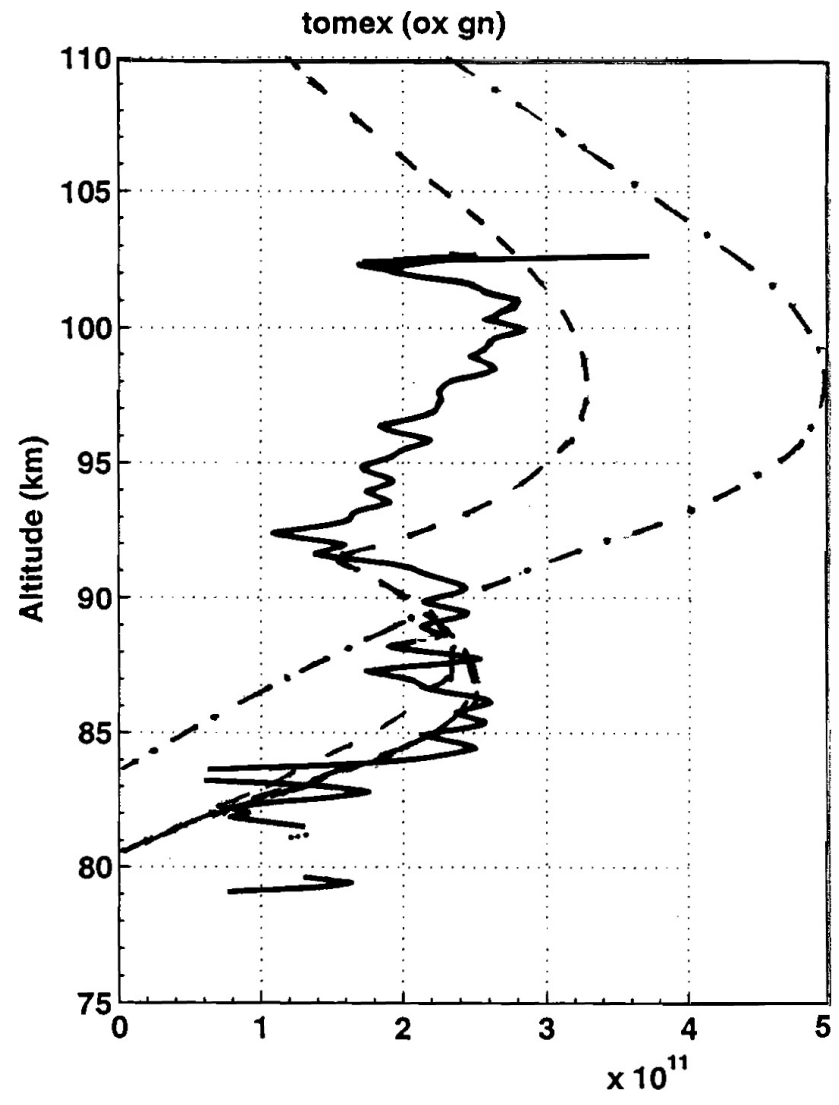

[O], $\mathrm{cm}^{-3}$

Fig. 11. Height profiles of atomic oxygen density inferred from the green line emission measured during the TOMEX (solid line), calculated by the model (dashed line), and given by the MSISE-90 model (dashed-dotted line).

ture maximum is located around the heating rate peak. Our model shows that the eddy coefficient peak must be at an altitude of $92 \mathrm{~km}$ to provide a temperature profile with the $86 \mathrm{~km}$ peak given by the TIMED-GCM model. Thus, a considerable difference appears to exist between the model described here and the one used for TIME-GCM, even when applied to their parameters. That is, the eddy diffusion properties in TIME-GCM do not seem to be consistent with their temperature profile. One of the main causes for the difference between our results and those of the TIME-GCM model may be significant uncertainty in the cooling rate in the mesosphere. However, using the eddy heat conductivity coefficient with the parameters $K_{\mathrm{ec}}^{m}=2.5 \times 10^{6} \mathrm{~cm}^{2} \mathrm{~s}^{-1}$ and $z_{m}=100 \mathrm{~km}$, which are close to the parameters used by the TIMED-GCM model, our model reproduces the temperature height profile with the $97 \mathrm{~km}$ peak measured by Hecht et al. (2004). This result can be seen from the measured and calculated profiles shown in Fig. 10.

The height profile of atomic oxygen density derived from the rocket-borne green line photometer data is shown in 
Fig. 11. This profile has two peaks at altitudes of $85 \mathrm{~km}$ and $100 \mathrm{~km}$ (Hecht et al., 2004). Using the TIME-GCM model, Hecht et al. (2004) tried to describe the observed height distributions of atomic oxygen and temperature. The model calculations showed that the $[\mathrm{O}]$ profile had only one peak at $90.5 \mathrm{~km}$ altitude.

Our model reproduces an [O] height profile with two peaks, shown in Fig. 11. In this case, the maximum of the eddy diffusion coefficient is located at an altitude of $92 \mathrm{~km}$ and the $K_{\text {ed }}$ maximum value is $2 \times 10^{6} \mathrm{~cm}^{2} \mathrm{~s}^{-1}$. As also shown above, the heating corresponding to this eddy turbulence can provide a temperature maximum of $230 \mathrm{~K}$, which is close to the $T$ maximum measured during the rocket experiment. These model results show that a very close connection exists between the temperature peak and atomic oxygen density.

Note that eddy turbulence heating occurs below the $K_{\mathrm{ec}}$ peak. These important features should be taken into account in estimating the eddy diffusion coefficient from data on the energy dissipation rate. For example, the eddy diffusion coefficients inferred by Lübken (1997) from energy dissipation rates should most likely be shifted to higher altitudes.

\section{Conclusions}

We studied heating and cooling in the upper mesosphere due to dissipation of gravity waves as described by eddy turbulence, eddy conductivity, chemical heating, and radiative cooling. Heating by eddy turbulence occurs at altitudes below the eddy diffusion coefficient peak. This feature should be taken into account when the eddy diffusion coefficient is inferred from data on turbulent energy dissipation. The eddy diffusion heating rate depends on the $K_{\text {ed }}$ maximum value and the $K_{\text {ed }}$ gradient below the peak. The role of both parameters is comparable, and an increase in heating is possible due to the $K_{\text {ed }}$ maximum increase and/or the $K_{\text {ed }}$ gradient increase. Also, the heating maximum depends on both parameters. The main cooling by eddy turbulence occurs above the $K_{\text {ed }}$ peak altitude and strongly increases with an increasing negative gradient in the $K_{\text {ed }}$ height distribution.

The eddy turbulence heating rate strongly depends on the Richardson number. Usually the temperature peak is located in the area corresponding to high wind shear. Dynamic instability needs a large wind shear because of the positive temperature gradient below the peak. We suggest that the wind shear may be strongest during equinox when the global change of atmospheric circulation occurs. As far as we know, seasonal variations of wind shear have not been published.

We also considered splitting of the atomic oxygen distribution peak, which is observed during the same period when the double mesopause is observed. According to our results, this splitting is produced at the altitude transitions from uniform eddy turbulence to localized turbulence and then to molecular diffusion, and the depth of the gap strongly depends on the negative gradient of the eddy diffusion coefficient above the $K_{\text {ed }}$ peak. Our results show that the eddy turbulence height distribution used to produce the double mesopause also can be used to produce an $[\mathrm{O}]$ height profile with small splitting. However, deep splitting also requires a steep negative gradient of the $K_{\text {ed }}$ height profile above the $K_{\text {ed }}$ peak to induce strong cooling above the temperature maximum in the upper mesosphere. The main result of this study is that eddy turbulence can provide the temperature peak (associated with the double mesopause) and the atomic oxygen double layer in the upper mesopause. This may explain why both phenomena are observed during the same period. We emphasize that more simultaneous measurements of the temperature peak and the $[\mathrm{O}]$ double layer in the upper mesosphere are needed to check our approach. At the same time, analyzing the experimental data based on this approach will be very important in understanding the main features of eddy turbulence and in solving the problem of uniform and localized turbulence in the MLT.

Acknowledgements. Research at Cornell University was supported by the National Science Foundation under grant ATM-0551107.

Topical Editor C. Jacobi thanks three anonymous referees for their help in evaluating this paper.

\section{References}

Akmaev, R. A.: On the energetics of mean-flow interactions with thermally dissipating gravity waves, J. Geophys. Res., 112, D11125, doi:10.1029/2006JD007908, 2007.

Banks, P. M. and Kockarts, G.: Aeronomy, Academic Press, 1973.

Becker, E.: Direct heating rates associated with gravity wave saturation, J. Atmos. Solar-Terr. Phys., 66, 683-696, 2004.

Becker, E. and McLandress, C.: Consistent scale interaction of gravity waves in the Doppler spread parameterization, J. Atmos. Sci., 66, 1434-1449, 2009.

Bills, R. E. and Gardner, C. S.: Lidar observations of the mesopause region temperature structure at Urbana, J. Geophys. Res., 98, 1011-1021, 1993.

Brasseur, G. and Solomon, S.: Aeronomy of the Middle Atmosphere, D. Reidel Publishing Company, Dordrecht, Holland, 1986.

Fomichev, V. I., Kutepov, A. A., Akmaev, R. A., and Shved, G. M.: Parameterization of the $15 \mu \mathrm{m} \mathrm{CO} 2$ band cooling in the middle atmosphere (15-115 km), J. Atmos. Terr., 55, 7-18, 1993.

Fritts, D. C. and Luo, Z.: Dynamical and radiative forcing of the summer mesopause circulation and thermal structure, 1. Mean solstice conditions, J. Geophys. Res., 100, 3119-3128, 1995.

Fukao, S., Yamanaka, M. D., Ao, N., Hocking, W. K., Sato, T., Yamamoto, M., Nakamura, T., Tsuda, T., and Kato, S.: Seasonal variability of vertical eddy diffusivity in the middle atmosphere, 1. Three-year observations by the middle and upper atmosphere radar, J. Geophys. Res., 99, 18973-18987, 1994.

Gordiets, B. F. and Kulikov, Yu. N.: Numerical modeling of the terrestrial thermosphere thermal structure, Kosm. Issled., 19, 249$260,1981$.

Gusev, O., Kaufmann, M., Grossmann, K.-U., Schmidlin, F. J., and Shepherd, M. G.: Atmospheric neutral temperature distribution 
at the mesopause altitude, J. Atmos. Solar-Terr. Phys., 68, 16841697, 2006.

Hecht, J. H., Liu, A. Z., Walterscheid, R. L., Roble, R. G., Larsen, M. F., and Clemmons, J. H.: Airglow emissions and oxygen mixing ratios from the photometer experiment on the Turbulent Oxygen Mixing Experiment (TOMEX), J. Geophys. Res., 109, D02S05, doi:10.1029/2002JD003035, 2004.

Hedin, A. E.: Extension of the MSIS thermosphere model into the middle and lower atmosphere, J. Geophys. Res., 96, 1159-1172, 1991.

Hocking, W. K.: Turbulence in the region 80-120 km, Adv. Space Phys., 10, 153-161, 1990.

Hocking, W. K.: The dynamical parameters of turbulence theory as they apply to middle atmospheric studies, Earth Planets Space, 51, 525-541, 1999.

Kelley, M. C., Kruschwitz, C. A., Gardner, C. S., Drummond, J. D., and Kane, T. J.: Mesospheric turbulence measurements from persistent Leonid meteor train observations, J. Geophys. Res., 108, 8454, doi:10.1029/2002JD002392, 2003.

Larsen, M. F.: Winds and shears in the mesosphere and lower thermosphere: Results from four decades of chemical release wind measurements, J. Geophys. Res., 107, 1215, doi:1029/2001JA000218, 2002.

Liu, G. and Shepherd, G. G.: Perturbed profiles of oxygen nightglow emissions as observed by WINDII on UARS, J. Atmos. Solar-Terr. Phys., 68, 1018-1028, 2006.

Lübken, F. J.: Seasonal variation of turbulent energy dissipation rates at high latitudes as determined by in situ measurements of neutral density fluctuations, J. Geophys. Res., 102, 1344113456, 1997.
Medvedev, A. S. and Klaassen, G. P.: Thermal effects of saturating gravity waves in the atmosphere, J. Geophys. Res., 108, 4040, doi:10.1029/2002JD002504, 2003.

Peixoto, J. P. and Oort, A. H.: Physics of Climate, American Institute of Physics, New York, 1992.

Shimazaki, T.: Effective eddy diffusion coefficient and atmospheric composition in the lower thermosphere, J. Atmos. Terr. Phys., 33, 1383-1401, 1971.

Smith, A. K., Marsh, D. F., and Szymczak, A. C.: Interaction of chemical heating and tides in the mesosphere, J. Geophys. Res., 108, 4164, doi:10.1029/2002JD002664, 2003.

Tohmatsu, T.: Compendium of Aeronomy, Kluwer Academic Publishers, Norwell, MA, 1990.

Vlasov, M. N. and Davydov, V. E.: Splitting of atomic oxygen height profiles by eddy diffusion, J. Atmos. Terr. Phys., 55, 1361-1363, 1993.

Vlasov, M. N. and Kelley, M. C.: Estimates of eddy turbulence consistent with seasonal variations of atomic oxygen and its possible role in the seasonal cycle of mesopause temperature, Ann. Geophys., 28, 2103-2110, doi:10.5194/angeo-28-2103-2010, 2010.

Vlasov, M. N. and Korobeynikova, T. V.: Effect of the turbulence, infrared radiation and mass-averaged transport on the height distribution of temperature in the middle-latitude and high latitude thermosphere, Cosmic Res., 29, 469-474, 1991.

Weinstock, J.: Vertical turbulent diffusion in a stably stratified fluid, J. Atmos. Sci., 35, 1022-1027, 1978.

Yu, J. R. and She, C. Y.: Climatology of a midlatitude mesopause region observed by a lidar at Fort Collins, Colorado $\left(40.6^{\circ} \mathrm{N}\right.$, $105^{\circ}$ W), J. Geophys. Res., 100, 7441-7452, 1995. 